\title{
A Noradrenaline-Induced Inhibition from Locus Coeruleus of Nucleus Accumbens Neuron Receiving Input from Hippocampus
}

\author{
Hiroshi UNEMOTO, Masashi SASA and Shuji TAKAORI \\ Department of Pharmacology. Faculty of Medicine. Kyoto University, Kyoto 606, Japan
}

Accepted July 6,1985

\begin{abstract}
Electrophysiological studies using rats anesthetized with chloral hydrate were performed to determine whether or not noradrenaline originating in the locus coeruleus (LC) produces a beta-receptor-mediated inhibition of the nucleus accumbens (AcC) neurons receiving input from the hippocampus (HPC). When conditioning stimuli were applied to the LC preceding a test stimulus to the HPC, an inhibition of spike generation with HPC stimulation was observed during 20-100 msec of the conditioning-test time interval. This inhibition was observed when the stimulating electrode was located in the LC or its immediate vicinity. The spike generation upon HPC stimulation was also inhibited by iontophoretic application of noradrenaline, and the inhibition was antagonized by iontophoretically applied sotalol, a beta-adrenergic blocker, but not by phentolamine, an alpha-adrenergic blocker. These results suggest that noradrenaline derived from the LC produces a beta-receptor-mediated inhibition of the Acc neurons receiving input from the HPC.
\end{abstract}

The nucleus accumbens (Acc), which is considered to be one of the functional interfaces between the limbic system and the motor cortex, receives inputs from several areas of the brain such as the hippocampus (HPC), amygdala, septum, olfactory tubercle. cingulate gyrus, caudate nucleus and thalamic nuclei (1-5). The Acc also receives dopaminergic innervation mainly from the ventral tegmental area and noradrenergic innervation presumably from the locus coeruleus (LC) $(6-10)$. Our previous studies demonstrated that dopamine originating in the ventral tegmental area inhibits the Acc neurons receiving input from the parafascicular nucleus of the thalamus, but not from the HPC $(11,12)$. In addition, we found that stimulation of the LC and microiontophoretically applied noradrenaline inhibited the Acc neurons receiving input from the HPC, but rarely from the parafascicular nucleus, and the $\mathrm{LC}$-induced inhibition was antagonized by sotalol, a beta-adrenergic blocker (13).

In the next step for demonstrating that noradrenaline originating in the LC produces a beta-receptor-mediated inhibition of the Acc neuron, we examined whether or not 1) the inhibitory effect of conditioning stimulation on the HPC-induced spikes of the Acc neurons can be detected only when the stimulating electrode was positioned in the LC, and 2) the effects of exogeneously applied noradrenaline on the Acc neurons are also antagonized by the beta-adrenergic blocker.

\section{Materials and Methods}

Seventy-three male Wistar rats weighing 300-500 g were anesthetized with chloral hydrate $(300 \mathrm{mg} / \mathrm{kg}$. i.p.). After tracheal cannulation, the animal was fixed in a stereotaxic instrument and a part of the skull was removed to allow insertion of electrodes. The animal was then immobilized with gallamine triethiodide $(80 \mathrm{mg} / \mathrm{kg}$, i.p.) and artificially respired. Body temperature was maintained at $36.5-37.5^{\circ} \mathrm{C}$ with a heating pad, and the ECG (Il lead) was continuously monitored during experiments.

Bipolar stimulating electrodes with a tip diameter of $0.1 \mathrm{~mm}$ and a tip separation of 
$0.1 \mathrm{~mm}$ were inserted, ipsilaterally into the $\mathrm{HPC}(\mathrm{A}, 3.0 ; \mathrm{L}, 4.5 ; \mathrm{H}, 0.0$ ) according to the brain atlas of König and Klippel (14). Test stimuli, composed of a square pulse $(0.2$ msec, $0.1-1.0 \mathrm{~mA}$ ) were applied to the HPC every $1.6 \mathrm{sec}$. Conditioning stimuli, composed of 4 train pulses $(0.2 \mathrm{msec}, 0.1-1.0$ $m A, 1.000 \mathrm{~Hz}$ ) were applied to the LC (AP. 8.5; L. 1.0; H. 6.5), according to the brain atlas of Fifková and Maršala (15), 10-200 msec preceding the test stimuli to the HPC. Single neuron activities were extracellularly recorded in the Acc (A. 9.0-10.5; L. 1.0$2.0 ; \mathrm{H}, 6.0-8.0 \mathrm{~mm}$ from cortical surface) using a glass microelectrode filled with $2 \mathrm{M}$ sodium acetate containing Fast Green FCF and an electrical resistance of $10-20 \mathrm{M} \Omega$.

A seven-barreled micropipette (outer diameter: $5 \mu \mathrm{m}$ ) attached along the recording microelectrode was used for the microiontophoretic study. The distance between tips of the recording microelectrode and the micropipette was $15-20 \mathrm{~km}$. Each micropipette was filled with $0.5 \mathrm{M}$ dl-noradrenaline bitartrate (Sigma, $\mathrm{pH} 4.5$ ), $0.5 \mathrm{M}$ sotalol hydrochloride (Mead Johnson, pH 6.0), 30 $\mathrm{mM}$ phentolamine mesylate (CIBA, $\mathrm{pH} 6.0$ ).

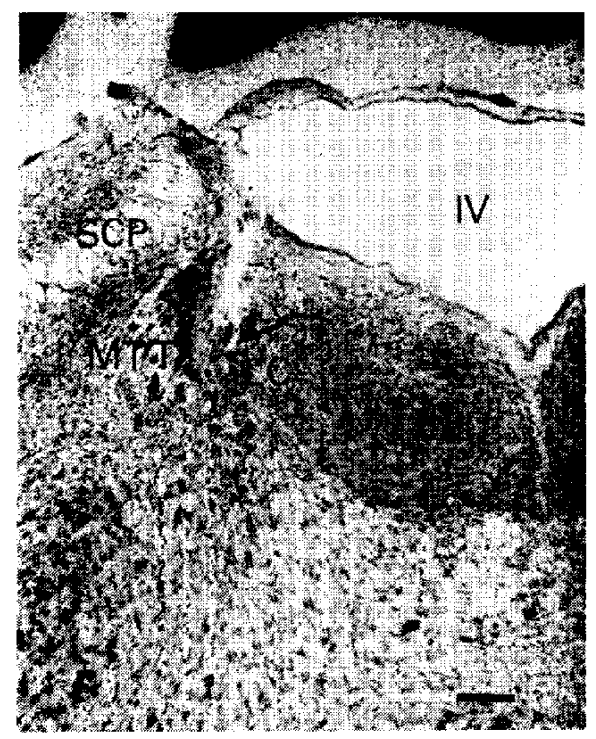

Fig. 1. The site of stimulating electrode (arrow) within the locus coeruleus (LC). Abbreviation MTT, mesencephalic tract of spinal trigeminal nerve SCP, superior cerebellar peduncle; IV, fourth ventricle. Calibration mark: $0.2 \mathrm{~mm}$.
$1 \mathrm{M}$ monosodium L-glutamate (Wako, $\mathrm{pH}$ 7.0) and $3 \mathrm{M} \mathrm{NaCl}$. These chemicals were iontophoretically applied to the immediate vicinity of the target neuron being recorded using a microiontophoresis programmer (WP-I, Model 160). A retaining current of 10-20 nA was applied to prevent the spontaneous release of the chemicals from the micropipette. Ten to 30 successive responses amplified and displayed on an oscilloscope (Nihon Kohden, VC-9) were photographed. The statistical significance of the data was determined by Student's $t$-test.

After the termination of each experiment. the stimulating and recording sites were marked by passing a direct current of 20-30 "A for 20-30 sec, and the locations were histologically checked with cresyl violet stain. The typical position of the stimulating electrode in the LC is shown in Fig. 1. Further details of the experimental procedures have been described previously $(12,13,16$, 17).

\section{Results}

1. Effects of LC conditioning stimulation: Figure 2 shows a typical example of an Acc neuron in which conditioning stimuli applied to the LC $30 \mathrm{msec}$ prior to the test stimulus to the HPC inhibited the spike generation upon HPC stimulation. Effects of the time interval between the conditioning and test stimulation ( $\mathrm{C}-\mathrm{T}$ interval) on the spike generation of 7 Acc neurons upon HPC stimulation are represented in Fig. 3. The inhibition was maximum at 20-30 $\mathrm{msec}$ and lasted for 100 msec of the $\mathrm{C}-\mathrm{T}$ interval.

Figure 4 is a schematic representation of the sites of the stimulating electrode tip in and outside of the LC region in 55 animals examined. When the stimulating electrode was located in the LC or the immediate vicinity of the LC, the inhibitory effect of conditioning stimulation on the HPCinduced spikes of the Acc neurons was observed in 22 animals. In 18 animals in which the electrode was positioned in the LC, however, alterations of the HPC-induced spikes were not obtained with conditioning stimulation in the Acc neurons tested. The conditioning stimulation had no significant effects on the HPC-induced spikes in any of 

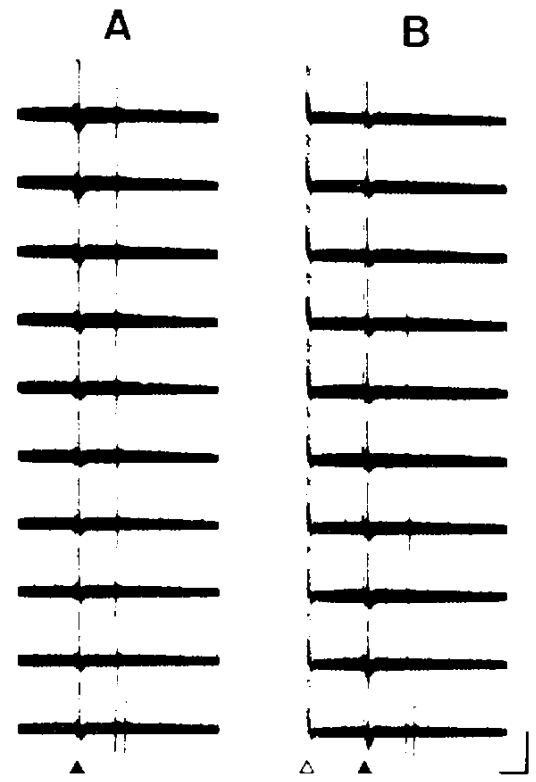

Fig. 2. Effects of conditioning stimulation of the locus coeruleus (LC) on spike generation of the nucleus accumbens neuron upon stimulation of hippocampus (HPC). A, control; B, with LC conditioning stimulation. Solid and open triangles indicate the stimuli artifacts to the HPC and $L C$. respectively. Calibration: $10 \mathrm{msec}, 1 \mathrm{mV}$.
15 animals in which the stimulating electrodes were located outside of the LC region such as the superior cerebellar peduncle, cerebellum or mesencephalic tract of the trigeminal nerve

2. Sotalol and phentolamine on noradrenaline-induced inhibition: When noradrenaline in a dose of $25 \mathrm{nA}$ was iontophoretically applied to the Acc neurons activated by HPC stimulation, the inhibition of the spike generation upon HPC stimulation was observed in 5-10 sec (compare Fig. 5A with $B$ ), and usually recovered $1.5-2.0 \mathrm{~min}$ after the termination of noradrenaline application.

Effects of sotalol and phentolamine were examined in 13 Acc neurons in which the HPC-induced spikes were significantly $(P<0.05)$ inhibited by a prior application of noradrenaline. Sotalol and phentolamine were iontophoretically applied for $1 \mathrm{~min}$ before the noradrenaline application. In the presence of sotalol (150 nA) and phentolamine $(150 \mathrm{nA})$. the spike generation produced by HPC stimulation remained unaffected (Fig. 5C and E). However, the inhibitory effect of noradrenaline application on the HPC-induced spikes was apparently reduced during iontophoretic application of

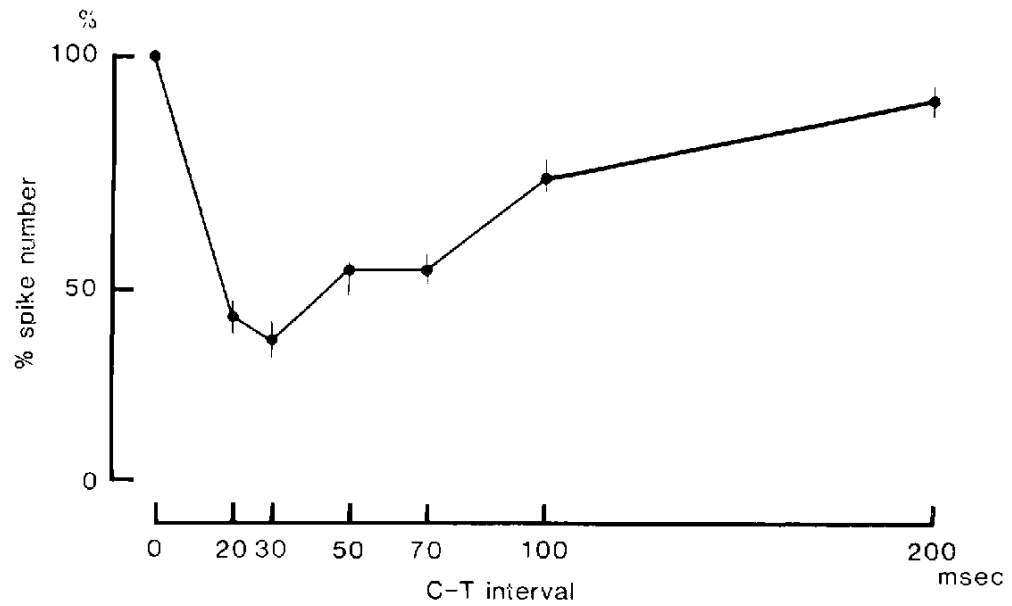

Fig. 3. Effects of time interval between conditioning stimulation of the locus coeruleus (LC) and test stimulation of the hippocampus (C-T interval) on spike generation of nuclelis accumbens neurons upon the test stimulation. The mean was obtained from 7 neurons, each of which was inhibited by the LC conditioning stimulation at a C-T interval of $30 \mathrm{msec}$ and could be successfully tested for the full time course. Vertical bars for each point indicate the standard error. 


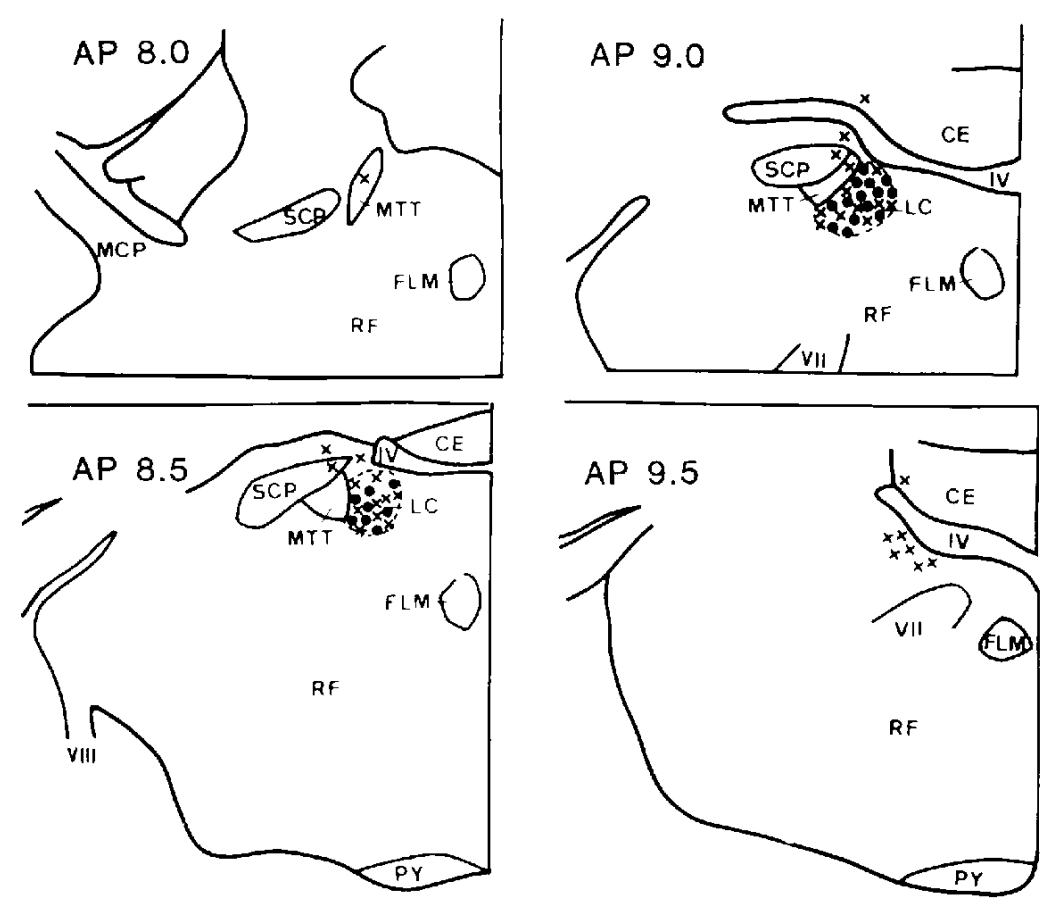

Fig. 4. Schematic representation of the location of stimulating electrodes in the region of the locus coeruleus (LC). AP 8.0-9.5 correspond to the frontal planes with references to the brain atlas of Fifková and Maršala (15). The effects of the conditioning stimulation were examined on 5-7 neurons in each animal. When the effect was obtained in at least one neuron, the stimulating site was judged as being effective. sites at which stimulation was effective in inhibiting the spike generation of nucleus accumbens neurons upon hippocampal stimulation; $x$, sites at which stimulation was ineffective. Abbreviations: CE. cerebellum: FLM. fasciculus longitudinalis medialis: MCP. medial cerebellar peduncle: MTT, mesencephalic tract of trigeminal nerve: PY, pyramides: RF, reticular formation; SCP. superior cerebellar peduncle; IV, fourth ventricle; VII, facial nerve; VIII, 8th cranial nerve.

sotalol (Fig. 5D). A marked antagonism was observed in 6 out of 13 neurons tested. When noradrenaline (25 $\mathrm{nA}$ ) was applied to the $\mathrm{Acc}$ neurons, the mean spike number upon HPC stimulation was significantly $(P<0.01)$ reduced from $1.00 \pm 0.94$ to $0.43 \pm 0.03$ $(n=13)$ (Table 1). The mean spike number was significantly increased to $0.64 \pm 0.04$ during sotalol application. compared with the value without sotalol. In contrast, the noradrenaline-induced inhibition of spikes upon HPC stimulation was not affected by the iontophoretic application of phentolamine in 12 out of 13 neurons tested (Fig. $5 \mathrm{~F}$ ). while the reduction of noradrenalineinduced inhibition by phentolamine was found in the remaining one neuron. The mean spike number of 13 neurons during simul- taneous application of noradrenaline (25 $\mathrm{nA})$ and phentolamine (150 nA) was $0.38 \pm 0.04$ (Table 1).

\section{Discussion}

The present studies confirmed our previous findings (13) that LC conditioning stimulation produced an inhibition of the Acc neuron receiving input from the HPC. This inhibition was observed only when the stimulating electrode was positioned in the LC or its immediate vicinity. These findings are in line with those that the inhibitory effects of conditioning stimulation on spinal trigeminal nucleus neurons are found when the stimulating electrode was located within or in the immediate vicinity of the $L C$ region (18). However, the effects of the con- 


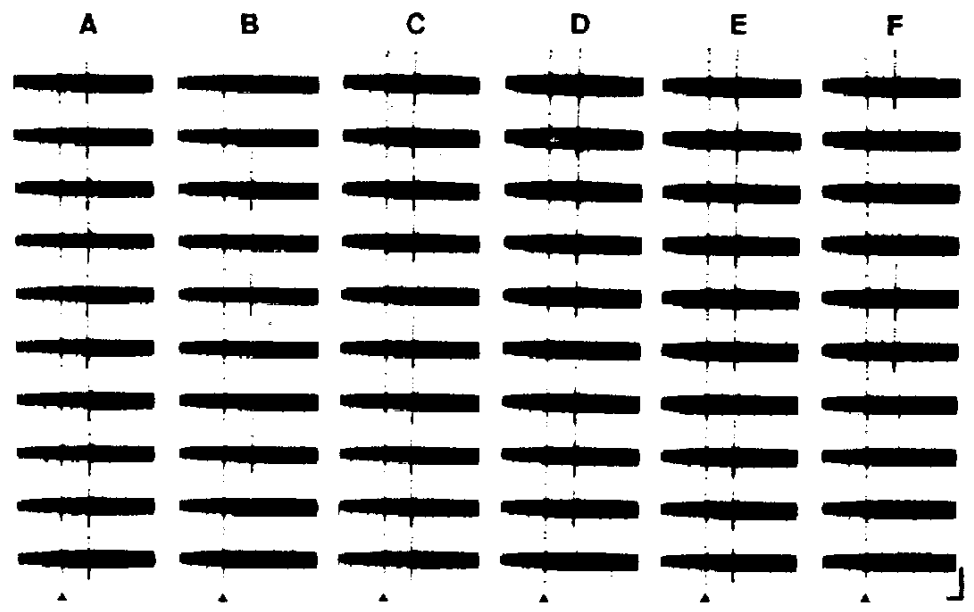

Fig. 5. Effects of sotalol (150 nA) and phentolamine (150 nA) on noradrenaline-induced inhibition of spikes in a nucleus accumbens neuron upon hippocampal stimulation. A, control; B, during iontophoretic application of noradrenalıne (25 nA): C. durıng iontophoretic application of sotalol: D. during simultaneous application of noradrenaline and sotalol: $\mathrm{E}$, during iontophoretic application of phentolamine: $F$, during simultaneous application of noradrenaline and phentolamine. Solid triangles indicate the stimulus artifacts to the hippocampus. Calibration: $10 \mathrm{msec}, 1 \mathrm{mV}$.

Table 1. Effects of iontophoretic noradrenaline (NA, $25 \mathrm{nA}$ ) on spikes elicited by hippocampal stimulation in nucleus accumbens neurons $(n=13)$ and those of iontophoretic sotalo! (150 nA) and phentolamine (150 $\mathrm{AA}$ ) on NA-induced inhibition of the spikes

$\begin{array}{lcc} & \text { No. of spikes } & \text { Latency (msec) } \\ \text { Control } & 1.00 \pm 0.04 & 16.17 \pm 0.94 \\ \text { Noradrenaline (NA) } & 0.43 \pm 0.03^{*} & 16.18 \pm 0.99 \\ \text { Sotalol alone } & 0.92 \pm 0.06 & 16.05 \pm 0.98 \\ \text { Sotalol+NA } & 0.64 \pm 0.04^{* *} & 16.13 \pm 0.99 \\ & (6 / 13)^{* * *} & \\ \text { Phentolamine alone } & 0.81 \pm 0.03 & 16.15 \pm 0.99 \\ \text { Phentolamine+NA } & 0.38 \pm 0.04 & 16.20 \pm 0.99 \\ & (1 / 13) & \end{array}$

Data show mean $=$ S.E. ${ }^{*}$ P<0.01: significantly different from the value of control. * P<0.01: significantly different from the value of NA without sotalol. **: Parentheses indicate the number of neurons significantly $(P<0.05)$ antagonized by sotatol or phentolamine vs. the total number of neurons tested.

ditioning stimulation were not obtained in 18 out of 40 animals, in which all stimulating electrodes were positioned in the $\mathrm{LC}$ or its immediate vicinity. These results are probably due to a minor innervation of ACC neurons from the LC, as discussed previously, because the Acc neurons unaffected by LC conditioning stimulation were also not affected by noradrenaline (13).

Our previous report demonstrated that the effects of noradrenaline microiontophoretically applied to the Acc neurons mimicked those of $\mathrm{LC}$ conditioning stimulation and that the HPC-induced spikes were inhibited by both noradrenaline application and $L C$ conditioning stimulation in the same Acc neuron, while most neurons unaffected by $L C$ conditioning stimulation remained unaltered by noradrenaline (13). Our previous report also demonstrated that the $\mathrm{LC}$ - 
induced inhibition of spike generation upon HPC stimulation was antagonized during iontophoretic application of sotalol, a betaadrenergic blocker, but not of phentolamine. an alpha-adrenergic blocker (13). Furthermore, the antagonism by sotalol of noradrenaline-induced inhibition of spikes elicited by HPC stimulation was found in the present study. These results indicate that noradrenaline released from the nerve terminals originating in the $\mathrm{LC}$ neuron produces an inhibition of the Acc neuron activated by HPC stimulation. It is noteworthy that noradrenaline derived from the $L C$ inhibits the $A c c$ neuron receiving input from the HPC and dopamine derived from the ventral tegmental area inhibits the neuron receiving input from the parafascicular nucleus of the thalamus (11-13).

The results that both noradrenaline- and LC-induced inhibitions of spikes elicited by HPC stimulation were antagonized by sotalo but not by phentolamine suggest that the inhibition was mediated by beta-adrenergic receptors in the Acc neuron. A blockade of noradrenaline - and /or LC-induced inhibition by beta-adrenergic blockers has been observed in cerebellar Purkinje cells, spinal trigeminal nucleus neurons, hippocampal cells and pyramidal tract cells in the cerebral cortex $(19-22)$.

In conclusion, it is strongly suggested that noradrenaline derived from the LC produces a beta-receptor-mediated inhibition of the Acc neurons receiving input from the HPC.

Acknowledgement: This work was supported in part by a Grant-in-Aid for Scientific Research from the Ministry of Education. Science and Culturc. Japan.

\section{References}

1 Mogenson, G.J., Jones, D.L. and Yim, C.Y.: From motivation to action: Functional interface between the limbic system and the motor system. Prog. Neurobiol. 14, 69-97 (1980)

2 Groenewegen, H.J., Becker, N.E.H.M. and Lohman, A.H.M.: Subcortical afferents of the nucleus accumbens septi in the cat, studied with retrograde axonal transport of horseradish peroxidase and bensbenzamide. Neuroscience 5, 1903-1916 (1980)

3 Groenewegen, H.J., Room, P., Witter, M.P. and Lohman, A.H.M.: Cortical afferents of the nucleus accumbens in the cat, studied with anterograde and retrograde transport technique. Neuroscience 7, 977-995 (1982)

4 Powell, E.W. and Leman, R.B.: Connection of the nucleus accumbens. Brain Res. 105, $389-$ 403 (1976)

5 Swanson, L.W. and Cowan. W.M.: A note on the connections and developmert of the nucleus accumbens. Brain Res. 92, $324-330$ (1975)

6 Chronister, R.B., Sikes, R.W., Wood, J. and DeFrance, J.F.: The pattern of termination of ventral tegmental afferents into nucleus accumbens: an anterograde HRP analysis. Neurosci. Lett. 17. 231-235 (1980)

7 Fallon, J.H. and Moore, R.Y.: Catecholamine innervation of the basal forebrain. IV. Topography of the dopamine projection to the basal forebrain and neostriatum. J. Comp. Neurol. 180, 545-580 (1978)

8 Moore, R.Y. and Bloom, F.E.: Central catecholamine nouron systems: Anatomy ard physiology of the norepinephrine and epinephrine systems. Annu. Rev. Neurosci. 2, 113-168 (1979)

9 Swanson, L.W. and Hartmen, B.K.: The central adrenergic system. An immunofluorescence study of the location of cell bodies and their efferent connections in the rat utilizing dopamine- $\beta$-hydroxylase as a marker. J. Comp. Neurol. 163,467-506 (1975)

10 Versteeg. D.H.G., van der Gugten, J., de Jong, W. and Palkovitz, M.: Regional concentrations of noradrenaline and dopamine in the rat brain. Brain Res. 113. 563-574 (1976)

11 Akaike, A., Sasa, M. and Takaori, S.: Inhibition from ventral tegmental area of nucleus accumbens neurons in the rat. Brain Res. 225 , 189-194(1981)

12 Akaike, A., Sasa, M. and Takaori, S.: Microiontophoretic studies of the dopaminergic inhibition from the ventral tegmental area to the nucleus accumbens neurors. J. Pharmacol. Exp. Ther. 229, 859-864 (1984)

13 Unemoto, H., Sasa, M. and Takaori, S.: Inhibition from socus coeruleus of nucleus accumbens neurons activated by hippocampal stimulation. Brain Res. 338, 376-379 (1985)

14 König, J.F.R. and Klippel, R.A.: The Rat Brain: A Stereotaxic: Atlas. Krieger. Huntington. N.Y. (1970)

15 Fifková, E. and Maršala, J.: Stereotaxic atlases for the cat. rabbit and rat. In Electrophysiological Methods in Biological Research. 3rd Edition. Edited by Bures. J.. Petrán. M. and Zachar, J.. p. 653-731. Academic Press. London (1967)

16 Igarashi, S., Sasa, M. and Takaori, S.: Feedback 
loop between locus coeruleus and spina trigeminal nucleus neurons responding to tooth pulp stimulation in the rat. Brain Res. Bull. 4, 75-83 (1979)

17 Sasa, M., Fujimoto, S., Igarashi, S., Munekiyo, K. and Takaori, S.: Microiontophoretic studies on noradrenergic inhibition from locus coeruleus of spinal trigemina: nucleus neurons. J. Pharmacol. Exp. Ther. 210, 311-315 (1979)

18 Sasa, M., Munekiyo, K., Ikeda, H. and Takaori, S.: Noradrenaline-mediated inhibition by locus coeruleus of spinal trigeminal neurons. Brain Res. 80, 443-460 (1974)

19 Hoffer, B.J., Siggins, G.R. and Bloom, F.E.: Studies on norepinephrine-containing afferents to Purkinje cells of rat cerebellum. II. Sensitivity of Purkinje celis to norepinephrine and related substances administered by microiontoptroresis. Brain Res. 25, 523-534 (1971)

20 Sasa, M., Munekiyo, K., Igarashi, S. and Takaori, S.: Antagonizing effects of $\beta$-adrenergic blockers on locus coeruleus-induced inhibition of trigeminal nucleus neurons. Japan. J. Pharmacol. 26, 519-525 (1976)

21 Segal, M. and Bloom, F.E.: The action of norepinephrine in the rat hippocampus. 11. Activation of the input pathway. Brain Res. 72, 99-114 (1974)

22 Stone, T.W: Pharmacology of pyramidal tract cells in the cerebral cortex. Noradrenaline and related substances. Naunyn Schmiedebergs Arch. Pharmacol. 278, 333-346 (1973) 